\title{
Exploring the Potential Mechanism of Zhengan Xifeng Decoction for ischemic stroke based on Network pharmacology and Molecular Docking
}

\section{Xiaodong Feng ( $\sim$ fxd0502@163.com )}

The First Affiliated Hospital of Henan University of Chinese Medicine https://orcid.org/0000-00032716-1827

\section{Yasu Zhang \\ Henan University of Chinese Medicine}

xiaomin liu

Henan University of Chinese Medicine https://orcid.org/0000-0002-4656-9302

Junzi Long

Henan University of Chinese Medicine

Qian Gao

Henan University of Chinese Medicine

Mengyang Pan

Henan University of Chinese Medicine

\section{Research}

Keywords: Zhengan Xifeng Decoction, ischemic stroke, Neuroprotection, Angiogenesis, Network pharmacology, Molecular Docking

Posted Date: February 1st, 2022

DOI: https://doi.org/10.21203/rs.3.rs-1288426/v1

License: (c) (1) This work is licensed under a Creative Commons Attribution 4.0 International License. Read Full License 


\section{Abstract}

Backgroud Ischemic stroke (IS) is an acute cerebrovascular incident that threatens public health. Zhengan Xifeng Decoction (ZXD), as a common herbal formula, has been widely applied in clinical practice for IS. The purpose of this study was to investigate the bioactive ingredients and potential pharmacological mechanisms of ZXD for IS based on network pharmacology and molecular docking.

Methods Chemical ingredients of ZXD were screened from TCMSP, BATMAN-TCM and the literature. Then, the targets of the chemical ingredients were predicted through STITCH and SwissTargetPrediction databases. IS-related targets were selected from DisGeNET, DrugBank, MalaCards and GeneCards databases. And, the String database and Cytoscape 3.8.2 software were used to construct the proteinprotein interaction (PPI) network. Kyoto Encyclopedia of Genes and Genomes (KEGG) and Gene Ontology (GO) enrichment analysis were analyzed in Metascape. At last, Molecular docking was carried out using Open Babel GUI, AutoDock 1.5.6 and Pymol.

Results 284 active ingredients involving 2353 putative targets were identified in ZXD, of which 1098 targets were linked to IS. A total of 20 core targets including MAPK3, MAPK1, and AKT1 were identified by analyzing PPI network. The biological process associated with IS were related to response to growth factor, apoptotic signaling pathway, MAPK cascade and neuron death. And KEGG enrichment included that Prolactin signaling pathway, VEGF signaling pathway and ErbB signaling pathway were closely correlated with neurogenesis, neurotransmission, synaptic plasticity vascular growth factor regulation, in IS. Finally, molecular docking revealed that core ingredients of Quercetin, Carvone and Asparamide had well-binding ability to core targets.

\section{Conclusion}

The study suggested that ZXD treated IS by modulating multiple targets and pathways. And, the results demonstrated that the mechanism of ZXD anti-IS may be related to the regulation of neuroprotection and angiogenesis through Prolactin signaling pathway, VEGF signaling pathway and the ErbB signaling pathway.

\section{Introduction}

Ischemic stroke (IS) is an acute cerebrovascular incident caused by disorder of the cerebral blood supply, which mainly causes ischemia, hypoxia, and even neuronal necrosis of brain tissue that leads to neurological deficits. Research has shown that IS is a neurological disorder with high morbidity, mortality, disability, and recurrence rates, which seriously endangers public physical and mental health [1]. Overproduction of free radicals, calcium overload, excitotoxicity and oxidative stress in cerebral ischemia and hypoxia may trigger cell necrosis or apoptosis, resulting in neuronal death in IS. In addition, the acute inflammatory response in cerebral ischemia leads to the blood-brain barrier (BBB) destruction, brain edema, and neuronal injuries [2,3]. However, the effectiveness of therapeutic drugs for IS is limited, making it important to investigate and develop new therapeutic agents. 
As a classical formula in Chinese medicine, Zhengan Xifeng Decoction (ZXD) with the effect of calming liver and suppressing hyperactive yang, extinguishing wind and dredging collaterals, is often used in clinical practice for stroke [4]. ZXD consists of 12 herbal medicines: Niuxi (Achyranthis Bidentatae Radix), Zheshi (Haematitum), Longgu (Fossilia OssiaMastodi), Muli (Ostreae Concha), Guiban (Testudinis Carapax et Plastrum), Tiandong (Asparagi radix), Yinchen (Artemisiae Scopariae Herba), Xuanshen (Scrophulariae Radix), Gancao (Glycyrrhizae Radix et Rhizoma), Chuanlianzi (Toosendan Fructus), Maiya (Hordei Fructus Germinatus), Baishao (Paeoniae Radix Alba). Clinical study found that ZXD was effective in regulating inflammatory responses, improving neurological function and increasing daily living ability in patients with IS [5]. Animal study showed that ZXD significantly reduced cerebral edema and infarct size, which contributed to improve behavioral impairment in MCAO rats, and it regulated the expression of caspase-3 to reduce nerve cell apoptosis in ischemic areas [6]. However, the mechanism of ZXD for IS has not been clarified.

With the rapid development of available biomedical data, new insights for drug discovery are being provided by systems biology and pharmacology. Network pharmacology is able to be reconstructed by integrating molecular networks with multidisciplinary, including biochemistry, bioinformatics, and systems biology to facilitate the mining of drug effect through novel network models of "multi-target, multi-effect, complex disease" [7]. In this study, we used the network pharmacology approaches including protein-protein interaction (PPI) network construction, network topology analysis, gene function analysis and molecular docking to reveal the mechanism of ZXD for IS. Flow chart is shown in Figure 1.

\section{Materials And Methods}

\section{Active ingredients and corresponding targets collection}

TCMSP (https://old.tcmsp-e.com/tcmsp.php) [8], BATMAN-TCM (http://bionet.ncpsb. org/batman-tcm/) [9] and the literature were used to screen the ingredients of ZXD. Ingredients that meet oral bioavailability $\geq 20 \%$, BBB $\geq-0.3$, drug-likeness $\geq 0.1$ or meet the Lipinski Rule of Five were selected [10], in addition, ingredients did not meet the above requirements and had pharmacological activity were also included. TCMSP, BATMAN-TCM, STITCH (http://stitch.embl.de/)[11] and Swiss TargetPrediction (http://www.swisstargetprediction.ch/)[12]databases were used to obtain the targets of ingredients.

\section{Targets collection for IS}

The IS related targets were obtained from DisGeNET (https:// www.disgenet.org/)[13], DrugBank (https://go.drugbank.com/), MalaCards (https:// www.malacards.org/)[14] and GeneCard (https://www.genecards.org/) databases, and then the targets were normalized using the UniProt database (https:// www. uniprot.org/) [15].

\section{PPI network construction}

The common targets of ZXD and IS were obtained through website of Venn (http://bioinformatics.psb.ugent.be/webtools/Venn/), and the common targets were uploaded to STRING 
(https://www.string-db.org/) to obtain the PPI network [16]. The PPI network data were imported into Cytoscape 3.8.2 software for visualization and topology analysis, and the central modules and key targets of the PPI network were obtained in Cytoscape using plug-ins MCODE and cytoHubba.

\section{Construction of ingredient-target-IS network}

The active ingredient-common target-IS network was constructed in Cytoscape software, and the topological analysis was performed to screen the key ingredients according to the value of degree, betweenness centrality and closeness centrality.

\section{GO and KEGG pathway enrichment analysis}

The GO analysis including biological processes (BC), molecular functions (MF) and cellular components (CC), and KEGG enrichment analysis were analyzed in Metascape database [17]. $P$-values $<0.01$ was considered to be significant. The results of top 20 with statistical significance were visualized as bubble plots in the bioinformatics platform(http://www.bioinformatics.com.cn/).

\section{Verification with Molecular Docking}

The 3D structures of key active ingredients were obtained from PubChem database (https://pubchem.ncbi.nlm.nih.gov/), and the 3D structures of key targets were retrieved from RCSB PDB database(https://www1.rcsb.org/). The original ligand and water molecule were removed from the receptor protein via using the PyMOL software. Molecular docking was conducted by using Autodock Tools 1.5.6, and the docking results were visualized in PyMOL. The lower of the binding energy score, the better of the binding performance.

\section{Results}

\section{The active ingredients and targets of ZXD}

A total of 284 ingredients and the corresponding 2353 targets were obtained. Among them, 20 ingredients of Niuxi (Achyranthis Bidentatae Radix), 1 ingredient of Zheshi (Haematitum), 5 ingredients of Longgu (Fossilia OssiaMastodi), 10 ingredients of Muli (Ostreae Concha), 18 ingredients of Guiban (Testudinis Carapax et Plastrum), 9 ingredients of Tiandong (Asparagi radix),33 ingredients of Yinchen (Artemisiae Scopariae Herba), 13 ingredients of Xuanshen (Scrophulariae Radix), 141 ingredients of Gancao (Glycyrrhizae Radix et Rhizoma), 9 ingredients of Chuanlianzi (Toosendan Fructus), 23 ingredients of Maiya (Hordei Fructus Germinatus), 26 ingredients of Baishao (Paeoniae Radix Alba). The information of the ingredients was shown in Additional file 1(Table 1). 
Table 1

The information of the ingredients of ZXD

Herb

Niuxi (Achyranthis Bidentatae Radix)

\section{Active ingredients}

poriferasta-7,22E-dien-3beta-ol, Inophyllum E, Spinasterol, DIBP, DBP, 28norolean-17-en-3-ol, berberine, coptisine, wogonin, oleanolic acid, delta 7stigmastenol, baicalein, epiberberine, beta-sitosterol, Stigmasterol, palmatine, beta-daucosterol_qt, betaine, Ecdysterone, inokosterone
Zheshi

(Haematitum)

Longgu (Fossilia

OssiaMastodi)

Muli (Ostreae

Concha)

Guiban

(Testudinis

Carapax et

Plastrum)

Tiandong

(Asparagi radix)

Yinchen

(Artemisiae

Scopariae Herba)
Ferric Oxide

Calcium Phosphate, Calcium Carbonate, Phosphorus pentoxide, Magnesium oxide, Ferric oxide

calcium carbonate, calcium phosphate, taurine, zinc, docosahexenoic acid, eicosapentaenoic acid, Calcium Sulphate, Ferric Oxide, Silicon, Aluminum

Aspartic Acid, Calcium Carbonate, Phenylalanine, glycine, Lanine, methionine, serine, isoleucine, threonine, glutamic acid, histidine, leucine, arginine,

Y-aminobutyric acid, cystine, proline, lysine, tyrosine

Stigmasterol, quercetin, Ferulic acid, diosgenin, Coniferol, beta-sitosterol, Asparagine, Aspafilioside A, 7-Methoxy-2-methyl isoflavone

Vanillin, Scopoletin, Scoparone, Salicylic Acid, Rhamnocitrin, quercetin, PCymene, Methyleugenol, Isorhamnetin, Isoarcapillin, Hyperin, Eupalitin, Hydroxyacetophenone, Genkwanin, Furaldehyde, Eupatolitin, Eugenol, Cirsilineol, Chlorogenic Acid, Carvone, Carene, Capillene, Caffeic Acid, betasitosterol, Beta-Elemene, Beta-Caryophyllene, Azelaic Acid, Artepillin C, Artepillin A, Areapillin, Alpha-Terpineol, Alpha-Humulene, 4'-Methylcapillarisin,

Xuanshen

(Scrophulariae

Radix

Sugiol, P-Methoxycinnamic Acid, P-hydroxycinnamic acid, oleic acid, Methylchavicol, L-Asparagine, Harpagide, cinnamic acid, beta-sitosterol, Aucubin, 6-0-methylcatalpol_qt, 6-0-Methyl Catalpol, (-)-Nissolin

Gancao

(Glycyrrhizae

Radix et

Rhizoma)
Xambioona, Vestitol, Urea, Uralenol-3-Methylether, Uralenin, Uralene, Umbelliferone, Tetrahydropalmatine, Tetrahydroharmine, Sitosterol, Sinapic Acid, Sigmoidin B, Shinpterocarpin, Shinflavanone, Schaftoside, Ruvoside, Rutin, Pinocembrin, Phaseollinisoflavan, Phaseolinisoflavan, Phaseol, Ononin, oleanolic acid, Odoratin, Nicotiflorin, Neoisoliquiritin, Narwedine, Monoolein, Monoammonium Glycyrrhizinate, Methylglyoxal, Medicarpin,

Mairin, Lupiwighteone, Liquoric Acid, Liquiritin, Liquiritigenin, Licorisoflavan A, Licoricone, Licoricidin, Licoisoflavone B, Licoisoflavone, Licoisoflavanone, Licofuranocoumarin, Licocoumarone, licochalcone G, licochalcone a, Licoarylcoumarin, Licoagroisoflavone, Licoagrocarpin, kanzonols W, Kanzonol Z, Kanzonol U, Kanzonol F, Kanzonol B, Jaranol, Isotrifoliol, Isoquercitrin, Isoorientin, Isoononin, Isoliquiritigenin, Isoliensinine, Isolicoflavonol, Isoglycyrol, Isobavachin, Inflacoumarin A, Inermine, icos-5-enoic acid, HMO, Hispidulin, Hispaglabridin B, Hedysarimcoumestan B, Gmelofuran, Glyzaglabrin, Glyyunnanprosapogenin D, Glypallichalcone, Glycyrrhizin, Glycyrrhizic Acid, Glycyrrhisoflavone, Glycyrrhisoflavanone, Glycyrrhetinic Acid, Glycyrol, Glycyrin, 
Glycycoumarin, Glyasperins M, glyasperin F, glyasperin D, Glyasperin C, glyasperin B, Gloeosteretriol, Glisoflavanone, Glepidotin B, Glepidotin A, Glabrone, Glabridin, Glabrene, Glabranin, Ganoderic Acid A, Gancaonin P-3'Methylether, Gancaonin M, Gancaonin L, Gancaonin I, Gancaonin H, Gancaonin G, Gancaonin F, Gancaonin B, Gancaonin A,

gadelaidic acid, formononetin, Ferulic Acid, Eurycarpin A, Euchrenone, Echinatin, Dimethyl Sebacate, DIBP, dehydroglyasperins C, DBP, Daidzein dimethyl ether, Corylifolinin, Castanin, Astragalin, Alpha-Trihydroxy Coprostanic Acid, 8Prenylwighteone, 7-Methoxy-2-methyl isoflavone, 7-hydroxy-2-methyl-3-phenylchromone, 7-Acetoxy-2-methylisoflavone, 6-Prenylluteolin, 6-prenylated eriodictyol, 6-Hydroxyrubiadin, 5,6,7,8-Tetrahydro-4-Methylquinoline, 4'-0Methylglabridin, 4'-Methoxyglabridin, 3'-Methoxyglabridin, 3-Hydroxyglabrol, 3'Hydroxy-4'-0-Methylglabridin, 3,3'-Dimethylquercetin, 2,4,4'-Trihydroxychalcone, 1-Methoxyphaseollidin, 18beta-Glycyrrhetinic Acid, 18alpha-Glycyrrhetinic Acid, (Z)-1-(2,4-dihydroxyphenyl)-3-phenylprop-2-en-1-one, (2R)-7-hydroxy-2-(4hydroxyphenyl)chroman-4-one

Chuanlianzi

(Toosendan Fructus)

Maiya (Hordei

Fructus Germinatus)
Torachrysone, Toosendanin, Stigmasterol, Myristic Acid, Methyl Tormentate, Medioresinol, Mandenol, linolenic acid, Ethyl linolenate,

a-tocopheryl quinone, vitamin E, Vitamin D, vitamin B, Tyrosol, Tyramine, Tryptanthrin, Tryptamine, Sucrose, Sterigmato-cystin, Sphaelactone A,

Spermine, Pyrethrin I, Putrescine, Lecithin, Hordenine, Heterodendrin, Gramine, Choline, Catalase, beta-sitosterol, Betaine, Adenine

Baishao (Paeoniae Radix Alba)

Tellimagrandin I, Strictinin, Satol, Pyrethrin li, Pyrethrin I, Phenol, Pentagalloylglucose, Pedunculagin, Palbinone, Paeonol, Paeonilactone C,

Paeonilactone B, Paeonilactone A, oleanolic acid, Methyl linolelaidate, Mairin, Lactiflorin, Gallotannin, Gallocatechin, Gallicacid, Epigallocatechin,

D-Catechin, DBP, Catechin, Casuarictin, beta-sitosterol

\section{IS targets and PPI network}

A total of 4154 targets related- IS were obtained, of which 1098 were duplicated with targets of ZXD (Figure 2). A total of 118 key nodes with 3096 edges were involved in PPI network (Figure 3). 4 subclusters were obtained from MCODE analysis (Figure 4). In addition, the 20 key targets were identified by CytoHubba (Figure 5).

\section{Ingredient-target-IS network}

The ingredient-target-IS network included 6544 edges, 1098 nodes of targets and 276 nodes of ingredients (Figure 6). The top 20 key ingredients were screened according to value of degree, betweenness centrality and closeness centrality based on topology analysis Additional file 1(Table 2). 
Table 2

List of key ingredients in the top 20

\begin{tabular}{|c|c|c|c|c|}
\hline Active ingredients & Herbs & Degree & $\begin{array}{l}\text { Betweenness } \\
\text { Centrality }\end{array}$ & $\begin{array}{l}\text { Closeness } \\
\text { Centrality }\end{array}$ \\
\hline Quercetin & $\begin{array}{l}\text { Yinchen (Artemisiae Scopariae } \\
\text { Herba), Tiandong (Asparagi } \\
\text { radix) }\end{array}$ & 178 & 0.018719476 & 0.386888014 \\
\hline Carvone & $\begin{array}{l}\text { Yinchen (Artemisiae Scopariae } \\
\text { Herba) }\end{array}$ & 159 & 0.011934583 & 0.375272926 \\
\hline Asparagine & Tiandong (Asparagi radix) & 148 & 0.006331886 & 0.368435155 \\
\hline Gramine & $\begin{array}{l}\text { Maiya (Hordei Fructus } \\
\text { Germinatus) }\end{array}$ & 125 & 0.005652462 & 0.364334923 \\
\hline Capillene & $\begin{array}{l}\text { Yinchen (Artemisiae Scopariae } \\
\text { Herba) }\end{array}$ & 121 & 0.004724727 & 0.350944359 \\
\hline Myristic Acid & Chuanlianzi (Toosendan Fructus) & 115 & 0.004189071 & 0.362414338 \\
\hline Tetrahydropalmatine & $\begin{array}{l}\text { Gancao (Glycyrrhizae Radix et } \\
\text { Rhizoma) }\end{array}$ & 112 & 0.005191466 & 0.364334923 \\
\hline Azelaic Acid & $\begin{array}{l}\text { Yinchen (Artemisiae Scopariae } \\
\text { Herba) }\end{array}$ & 109 & 0.003631769 & 0.360703043 \\
\hline Tryptanthrin & $\begin{array}{l}\text { Maiya (Hordei Fructus } \\
\text { Germinatus) }\end{array}$ & 104 & 0.005202398 & 0.363564252 \\
\hline Phenylalanine & $\begin{array}{l}\text { Guiban (Testudinis Carapax et } \\
\text { Plastrum) }\end{array}$ & 90 & 0.003438173 & 0.356217617 \\
\hline Vitamin B & $\begin{array}{l}\text { Maiya (Hordei Fructus } \\
\text { Germinatus) }\end{array}$ & 83 & 0.003090385 & 0.356033143 \\
\hline Vitamin E & $\begin{array}{l}\text { Maiya (Hordei Fructus } \\
\text { Germinatus) }\end{array}$ & 76 & 0.002434101 & 0.349162011 \\
\hline Salicylic Acid & $\begin{array}{l}\text { Yinchen (Artemisiae Scopariae } \\
\text { Herba) }\end{array}$ & 63 & 0.002092352 & 0.355113636 \\
\hline $\begin{array}{l}\text { Alpha-Trihydroxy } \\
\text { Coprostanic Acid }\end{array}$ & $\begin{array}{l}\text { Gancao (Glycyrrhizae Radix et } \\
\text { Rhizoma) }\end{array}$ & 59 & 0.001366897 & 0.347925101 \\
\hline Tyramine & $\begin{array}{l}\text { Maiya (Hordei Fructus } \\
\text { Germinatus) }\end{array}$ & 57 & 0.001039679 & 0.341191067 \\
\hline Methionine & $\begin{array}{l}\text { Guiban (Testudinis Carapax et } \\
\text { Plastrum) }\end{array}$ & 52 & 0.000822594 & 0.339673913 \\
\hline \multirow[t]{2}{*}{ Carene } & $\begin{array}{l}\text { Yinchen (Artemisiae Scopariae } \\
\text { Herba) }\end{array}$ & 52 & 0.000989777 & 0.347749115 \\
\hline & Baishao (Paeoniae Radix & 51 & & \\
\hline
\end{tabular}




\begin{tabular}{|lllll|} 
Beta-Sitosterol & $\begin{array}{l}\text { Alba), Maiya (Hordei Fructus } \\
\text { Germinatus), Niuxi (Achyranthis } \\
\text { Bidentatae Radix), Tiandong } \\
\text { Asparagi radix) Xuanshen } \\
\text { Scrophulariae } \\
\text { Radix), Yinchen (Artemisiae } \\
\text { Scopariae Herba) }\end{array}$ & 0.001844805 & 0.352564103 \\
\hline Beta-Elemene & $\begin{array}{l}\text { Yinchen (Artemisiae Scopariae } \\
\text { Herba) }\end{array}$ & 51 & 0.000827316 & 0.345303867 \\
\hline Beta-Caryophyllene & $\begin{array}{l}\text { Yinchen (Artemisiae Scopariae } \\
\text { Herb) }\end{array}$ & 51 & 0.00068119 & 0.342210055 \\
\hline
\end{tabular}

\section{GO and KEGG pathway analysis}

GO enrichment analysis of 118 core targets obtained 209 potential BP, 89 CC and 79 MF, and the BP were mainly involved in response to growth factor, apoptotic signaling pathway, MAPK cascade and neuron death. The $\mathrm{CC}$ were mainly involved in transcription regulator complex, membrane raft, membrane microdomain and cytoplasmic side of membrane. And the MF were mainly involved in kinase binding, protein kinase binding, transcription factor binding and DNA-binding transcription factor binding. The top 20 terms were shown in Figure 7. KEGG enrichment analysis of 118 core targets obtained 126 pathways, mainly including Prolactin signaling pathway, VEGF signaling pathway, ErbB signaling pathway. The top 20 terms were shown in Figure 8. In addition, MCODE analysis of the PPI network obtained 4 subnetworks, and the results of KEGG pathway enrichment of sub-networks mainly included Prolactin signaling pathway, Neurotrophin signaling pathway, ErbB signaling pathway. (Figure 9).

\section{Molecular docking}

The MAPK1, MAPK2 and AKT1 of the key targets were selected to molecularly docked with the Quercetin, Carvone and Asparamide of the key ingredients. The results showed the key ingredients were well-bind to the active sites of three key targets. The docking results of key ingredients with the key targets were shown in Table 3, Figure 10.

Table 3

Docking results of key active ingredients with

key targets

\begin{tabular}{|llll|}
\hline \multirow{2}{*}{ Ingredients } & \multicolumn{3}{c|}{ Binding energy(KJ/mol) } \\
\cline { 2 - 4 } & MAPK3 & MAPK1 & AKT1 \\
\hline Quercetin & -16.9 & -22.2 & -18.3 \\
\hline Carvone & -23.1 & -27.0 & -23.8 \\
\hline Asparamide & -11.1 & -13.9 & -16.9 \\
\hline
\end{tabular}

\section{Discussion}


As one of the most common cerebrovascular diseases, the pathogenesis of IS is complicated [18]. At present, ZXD was found to have positive influences on reducing NIHSS scores in patients with IS. In addition, ZXD had the active effect on reducing brain edema and improving vascular endothelial function by inhibiting the overexpression of ET-1, TNF- $a$ and local inflammatory response [19]. The network pharmacology revealed that ZXD exerted the anti-IS effect by modulating multi-targets and pathways.

The key ingredients such as quercetin, carvone, and asparagine have been proven to be effective in alleviating brain damage and ameliorating neurological functions. Acidosis-induced activation of ASIC1a (acid-sensing ion channel 1a) facilitated intracellular calcium overload during IS, which was closely related to ischemic neuronal death [20]. Pandey et al. indicated that quercetin from Yinchen (Artemisiae Scopariae Herba) and Tiandong (Asparagi radix) exerted neuroprotective effects by modulating ASIC1a to reduce infarct volume and improve neurobehavior in IS [20]. In addition, quercetin glucoside had the curative potential for facilitating angiogenesis in ischemic tissues, which was essential for improving blood supply to ischemic areas and restoring neurological function after IS [21]. Carvone, an important ingredient of Yinchen (Artemisiae Scopariae Herba), was found to decrease infarct volume, alleviate neuroinflammation in cerebral ischemic tissues, and ameliorate neurological deficits of MCAO rats through downregulating the expression of NLRP3, caspase-1, TNF- $\alpha$ and IL-1 $\beta$ [22]. Asparagine from Tiandong (Asparagi radix) was crucial for nervous system balance, myelin formation and brain development [23]. Moreover, Asparagine-linked (N-linked) glycan chains of glycoprotein played an important role in angiogenesis [24].

From the analysis of the PPI network, MAPK3, MAPK1 and AKT1 of the key targets had critical effects on IS. MAPK3 (ERK1) and MAPK1 (ERK2) were important components of MAPK family that mediated cell growth, adhesion, survival and angiogenesis. The MEK/ERK signaling pathway was activated during IS, which enhanced the expression of TNF-a, IL-1ß, IL- 6 and iNOS in the cerebral vasculature, resulting in the destruction of the BBB, blockage of nerve cell migration and inhibition of neurogenesis and synaptogenesis $[25,26]$. Meng et al [27] found that Xuanshen (Scrophulariae Radix) from ZXD possibly inhibited neuronal apoptosis during IS by regulating the MAPK pathway which participated the stimulation of hypoxia-inducible factors in angiogenesis [28]. Akt, as a serine/threonine protein kinase, could exert the effect of pro-survival, anti-apoptotic activities and angiogenesis [29]. The studies concluded that early activation of Akt improved the oxygen supply/depletion balance in microregions after cerebral ischemia/ reperfusion. Moreover, AKT was critical to regulate the expression of VEGF that was essential for neurogenesis and angiogenesis after IS [30, 31]. Lei et al found that quercetin exerted neuroprotective effect by reducing ROS overproduction induced by cerebral ischemia and reperfusion in the hippocampus, which was probably related to the upregulation of Akt signaling [32].

BP analysis of the core targets mainly included response to growth factor, apoptotic signaling pathway and neuron death. Nerve growth factor had neurotrophic and anti-apoptotic effects, and vascular endothelial growth factor also directly affected neuronal growth, survival, and axonal growth in the nervous system, which might be essential in the treatment of IS. Moreover, the KEGG analysis indicated 
that the sub-networks were mainly related to neural regeneration, repair and angiogenesis, which were consistent with the results of BP analysis.

The enrichment analysis of KEGG showed that ZXD affected multiple pathways which had crucial roles in the treatment of IS, including Prolactin signaling pathway, VEGF signaling pathway and ErbB signaling pathway. Prolactin was a peptide hormone with biological functions involved in brain and behavior regulation. Glutamate toxicity led to excess $\mathrm{Na}^{+}$and $\mathrm{Ca}^{2+}$ influx into neuronal cells which was critical mechanism of neuronal death in IS [33]. The study proved that prolactin was involved in hypothalamic neuronal signaling through regulating the MAPK/ERK pathway, and was a potential candidate for neurotherapeutics as it significantly reduced brain calcium and nitrate levels in different brain regions of MCAO rats to improve cerebral ischemia-reperfusion injury [34,35]. Lin et al [36] found that quercetin might promote prolactin receptor (PRLR) expression through the activation of AKT which contributed to axon regeneration. VEGF was an angiogenic factor and a powerful neurogenic growth factor, which was able to stimulate neurogenesis in the subventricular zone and dentate gyrus of the hippocampus [37]. Liang et al showed that PI3K/AKT and MAPK/ERK pathways mediated VEGF transcription to promote angiogenesis in MCAO rats. In addition, administration of VEGF to MCAO rats induced angiogenesis of the ischemic border and improved neurobehavioral scores [38, 39]. Han et al found that tetrahydropalmatine, an ingredient of Gancao (Glycyrrhizae Radix et Rhizoma) in ZXD, alleviated ischemia-reperfusion injury by increasing the expression of VEGF [40]. NRG/ErbB signaling pathway was associated with axon formation, neurotransmission and synaptic plasticity. Noll et al discovered that NRG1, a ligand for ERbB3 and ERbB4, attenuated neuronal damage after IS, which was a potential target for the therapy of patients with stroke [41]. Thus, ZXD probably protected the brain from IS through neuroprotection and angiogenesis, which were mediated through interactions with the key targets and pathways that discussed above.

The study of network pharmacology revealed the putative key ingredients, targets, pathways and pharmacological mechanisms of ZXD for IS, which provided direction and basis for future research. However, there were probably some limitations in this study. Higher quality TCM databases were needed to ensure the accuracy and completeness of data. In addition, further biological studies were needed to validate the results of the above study.

\section{Conclusion}

In conclusion, we investigated the potential mechanism of ZXD in the treatment of IS based on network pharmacology approaches. The underlying functional ingredients such as quercetin, carvone, and asparagine were identified in this study which may exert the effect of neuroprotective and angiogenesis on IS through Prolactin signaling pathway, VEGF signaling pathway and ErbB signaling pathway. Furthermore, the results of molecular docking supported the conclusions. Our results provided a new insight to reveal the mechanism of herbal formulas of ZXD for IS.

\section{Declarations}




\section{Funding}

The study supported by Foundation of He'nan Educational Committee (ID:18A360012), the key scientific and technological project of Henan Province (ID: 192102310424), and National Natural Science Foundation of China (ID: 81704135).

\section{Availability of data}

All data are available in the manuscript and they are showed in figures and tables.

\section{Ethics approval and consent to participate}

Not applicable.

\section{Consent for publication}

Not applicable.

\section{Competing interests}

The authors declare no conflict of interest, financial or otherwise.

\section{Author contributions}

Yasu Zhang and Xiaomin Liu are equally contributor to this manuscript. study concept or design $₫$ Yasu Zhang; Xiaomin Liu; Xiaodong Feng; data collection: Junzi Long; data analysis or interpretation: Qian Gao; Mengyang Pan; Zhuoyan Gu; revision of the manuscript and study supervision: Xiaodong Feng. The author(s) read and approved the final manuscript.

\section{Author details}

${ }^{1}$ School of Rehabilitation Medicine, Henan University of Chinese Medicine,

Zhengzhou, China. ${ }^{2}$ The First Affiliated Hospital of Henan University of Chinese Medicine, Zhengzhou, China. "Ya-Su Zhang and Xiao-Min Liu contributed equally to this work.

\section{References}


1. GBD 2016 Neurology Collaborators. Global, regional, and national burden of neurological disorders, 1990-2016: a systematic analysis for the Global Burden of Disease Study 2016. Lancet Neurol. 2019;18:459-80. doi:10.1016/S1474-4422(18)30499-X.

2. Sekerdag E, Solaroglu I, Gursoy-Ozdemir Y. Cell Death Mechanisms in Stroke and Novel Molecular and Cellular Treatment Options. Curr Neuropharmacol. 2018;16:1396-415. doi:10.2174/1570159X16666180302115544.

3. Radak D, Katsiki N, Resanovic I, Jovanovic A, Sudar-Milovanovic E, Zafirovic S, et al. Apoptosis and Acute Brain Ischemia in Ischemic Stroke. Curr Vasc Pharmacol. 2017;15:115-22. doi:10.2174/ 1570161115666161104095522.

4. Xie XJ, Xing ZH, Zhu HX, An L. Effects of Zhengan Xifengtang on IL-6 and TNF-a in Patients with Diabetes Mellitus Complicated with Acute Cerebral Infarction. Chinese Journal of Experimental Traditional Medical Formulae. 2017;23:159-63. doi:10.13422/j.cnki.syfjx.2017020159.

5. Zhang S, Chou LX. Clinical Effect, Neurological Function and Prognosis of Patients with Diabetes Mellitus Complicated with Cerebral Infarction Treated with Zhengan Xifeng Decoction. Nursing Science. 2019;8:88-93. doi:10.12677/NS.2019.82018.

6. Wu YX, Wu TY, Ye H, Fu L. Effect of Zhengan Xifeng Decoction Pretreatment on Pathological Changes and Neuron Apoptosis of Cerebral Tissue in Rat, Chinese Journal of Experimental Traditional Medical Formulae. 2013; 224-228. doi:CNKI: SUN: ZSFX.0.2013-09-064.

7. Zhang GB, Li QY, Chen QL, Su SB. Network pharmacology: a new approach for chinese herbal medicine research. Evid Based Complement Alternat Med. 2013; 621423. doi:10.1155/2013/621423.

8. Ru J, Li P, Wang J, Zhou W, Li B, Huang C, et al. TCMSP: a database of systems pharmacology for drug discovery from herbal medicines. J Cheminform. 2014;6:13. doi:10.1186/1758-2946-6-13.

9. Liu Z, Guo F, Wang Y, Li C, Zhang X, Li H, et al. BATMAN-TCM: a Bioinformatics Analysis Tool for Molecular mechANism of Traditional Chinese Medicine. Sci Rep. 2016;6:21146. doi:10.1038/srep21146.

10. Lipinski CA, Lombardo F, Dominy BW, Feeney PJ. Experimental and computational approaches to estimate solubility and permeability in drug discovery and development settings. Adv Drug Deliv Rev. 2001;46:3-26. doi:10.1016/s0169-409x(00)00129-0.

11. Kuhn M, von Mering C, Campillos M, Jensen LJ, Bork P. STITCH: interaction networks of chemicals and proteins. Nucleic Acids Res. 2008;36:D684-8. doi:10.1093/nar/gkm795.

12. Daina A, Michielin O, Zoete V. SwissTargetPrediction: updated data and new features for efficient prediction of protein targets of small molecules. Nucleic Acids Res. 2019;47:W357-64. doi:10.1093/nar/gkz382.

13. Piñero J, Ramírez-Anguita JM, Saüch-Pitarch J, Ronzano F, Centeno E, Sanz F, et al. The DisGeNET knowledge platform for disease genomics: 2019 update. Nucleic Acids Res. 2020;48:D845-55. doi:10.1093/nar/gkz1021.

14. Rappaport N, Twik M, Plaschkes I, Nudel R, Iny Stein T, Levitt J, et al. MalaCards: an amalgamated human disease compendium with diverse clinical and genetic annotation and structured search. 
Nucleic Acids Res. 2017;45:D877-87. doi:10.1093/nar/gkw1012. Epub 2016 Nov 28.

15. UniProt Consortium. UniProt: the universal protein knowledgebase in 2021. Nucleic Acids Res. 2021;49:D480-9. doi:10.1093/nar/gkaa1100.

16. Szklarczyk D, Gable AL, Lyon D, Junge A, Wyder S, Huerta-Cepas J, et al. STRING v11: protein-protein association networks with increased coverage, supporting functional discovery in genome-wide experimental datasets. Nucleic Acids Res. 2019;47:D607-13. doi:10.1093/nar/gky1131.

17. Zhou Y, Zhou B, Pache L, Chang M, Chang M, Khodabakhshi AH, et al. Metascape provides a biologist-oriented resource for the analysis of systems-level datasets. Nat Commun. 2019;10:1523. doi:10.1038/s41467-019-09234-6.

18. Chen J, Venkat P, Zacharek A, Chopp M. Neurorestorative therapy for stroke. Front Hum Neurosci. 2014;8:382. doi:10.3389/fnhum.2014.00382.

19. Chen LF, Li JA, Zhang CY. Therapeutic effect of Zhengan Xifeng Decoction on acute ischemic stroke and vascular endothelial function. Journal Of China Prescription Drug. 2019;17:143-4. doi:CNKI:SUN:ZGCF.0.2019-09-086.

20. Pandey AK, Hazari PP, Patnaik R, Mishra AK. The role of ASIC1a in neuroprotection elicited by quercetin in focal cerebral ischemia. Brain Res. 2011;1383:289-99. doi:10.1016/j.brainres.2011.01.085.

21. Yin KJ, Hamblin M, Chen YE. Angiogenesis-regulating microRNAs and Ischemic Stroke. Curr Vasc Pharmacol. 2015;13:352-65. doi:10.2174/ 1570161111311999 0016.

22. Dai M, Wu L, Yu K, Xu R, Wei Y, Chinnathambi A, Alahmadi TA, Zhou M. D-Carvone inhibit cerebral ischemia/reperfusion induced inflammatory response TLR4/NLRP3 signaling pathway. Biomed Pharmacother. 2020;132:110870. doi:10.1016/j.biopha.2020.110870.

23. Newburg DS, Fillios LC. Brain development in neonatal rats nursing asparagine-deprived dams. Dev Neurosci. 1982;5:332-44. doi:10.1159/000112693.

24. Baksi K, Tavárez-Pagán JJ, Martínez JA, Banerjee DK. Unique structural motif supports mannosylphospho dolichol synthase: an important angiogenesis regulator. Curr Drug Targets. 2008;9:262-71. doi:10.2174/138945008783954916.

25. Maddahi A, Edvinsson L. Cerebral ischemia induces microvascular pro-inflammatory cytokine expression via the MEK/ERK pathway. J Neuroinflammation. 2010;7:14. doi:10.1186/1742-2094-714.

26. Yang K, Cao F, Sheikh AM, Malik M, Wen G, Wei H, et al. Up-regulation of Ras/Raf/ERK1/2 signaling impairs cultured neuronal cell migration, neurogenesis, synapse formation, and dendritic spine development. Brain Struct Funct. 2013;218:669-82. doi:10.1007/s00429-012-0420-7.

27. Meng X, Xie W, Xu Q, Liang T, Xu X, Sun G, et al. Neuroprotective Effects of Radix Scrophulariae on Cerebral Ischemia and Reperfusion Injury via MAPK Pathways. Molecules. 2018;23:2401. doi:10.3390/molecules23092401.

28. Liang Z, Chi YJ, Lin GQ, Luo SH, Jiang QY, Chen YK. MiRNA-26a promotes angiogenesis in a rat model of cerebral infarction via PI3K/AKT and MAPK/ERK pathway. Eur Rev Med Pharmacol Sci. 
2018; 22: 3485-92. doi: 10.26355/ eurrev_201806_15175.

29. Downward J. Mechanisms and consequences of activation of protein kinase B/Akt. Curr Opin Cell Biol. 1998;10:262-7. doi:10.1016/s0955-0674(98)80149-x.

30. Weiss HR, Chi OZ, Kiss GK, Liu X, Damito S, Jacinto E. Akt activation improves microregional oxygen supply/consumption balance after cerebral ischemia-reperfusion. Brain Res. 2018;1683:48-54. doi:10.1016/j.brainres.2018.01.019.

31. Ackah E, Yu J, Zoellner S, Iwakiri Y, Skurk C, Shibata R, et al. Akt1/protein kinase Balpha is critical for ischemic and VEGF-mediated angiogenesis. J Clin Invest. 2005;115:2119-27. doi:10.1172/JCl24726.

32. Lei X, Chao H, Zhang Z, Lv J, Li S, Wei H, et al. Neuroprotective effects of quercetin in a mouse model of brain ischemic/reperfusion injury via anti-apoptotic mechanisms based on the Akt pathway. Mol Med Rep. 2015;12:3688-96. doi:10.3892/mmr.2015.3857.

33. Choi DW. Excitotoxicity. Still Hammering the Ischemic Brain in 2020. Front Neurosci. 2020;14:579953. doi:10.3389/fnins.2020.579953.

34. Vermani B, Mukherjee S, Kumar G, Patnaik R. Prolactin attenuates global cerebral ischemic injury in rat model by conferring neuroprotection. Brain Inj. 2020;34:685-93.

doi:10.1080/02699052.2020.1726466.

35. Blume A, Torner L, Liu Y, Subburaju S, Aguilera G, Neumann ID. Prolactin activates mitogen-activated protein kinase signaling and corticotropin releasing hormone transcription in rat hypothalamic neurons. Endocrinology. 2009;150:1841-9. doi:10.1210/en.2008-1023.

36. Lin M, Wang N, Yao B, Zhong Y, Lin Y, You T. Quercetin improves postpartum hypogalactia in milkdeficient mice via stimulating prolactin production in pituitary gland. Phytother Res. 2018;32:151120. doi:10.1002/ptr.6079.

37. Jin K, Zhu Y, Sun Y, Mao XO, Xie L, Greenberg DA. Vascular endothelial growth factor (VEGF) stimulates neurogenesis in vitro and in vivo. Proc Natl Acad Sci USA. 2002;99:11946-50. doi:10.1073/pnas.182296499.

38. Liang Z, Chi YJ, Lin GQ, Luo SH, Jiang QY, Chen YK. MiRNA-26a promotes angiogenesis in a rat model of cerebral infarction via PI3K/AKT and MAPK/ERK pathway. Eur Rev Med Pharmacol Sci. 2018; 22: 3485-92. doi: 10.26355/eurrev _201806_15175.

39. Yang JP, Liu HJ, Liu XF. VEGF promotes angiogenesis and functional recovery in stroke rats. J Invest Surg. 2010;23:149-55. doi:10.3109/08941930903469482.

40. Han Y, Zhang W, Tang Y, Bai W, Yang F, Xie L, et al. I-Tetrahydropalmatine, an active component of Corydalis yanhusuo W.T. Wang, protects against myocardial ischaemia-reperfusion injury in rats. PLoS One. 2012; 7: e38627. doi: 10.1371/ journal.pone.0038627.

41. Noll JM, Li Y, Distel TJ, Ford GD, Ford BD. Neuroprotection by Exogenous and Endogenous Neuregulin-1 in Mouse Models of Focal Ischemic Stroke. J Mol Neurosci. 2019;69:333-42. doi:10.1007/s12031-019-01362-4. 

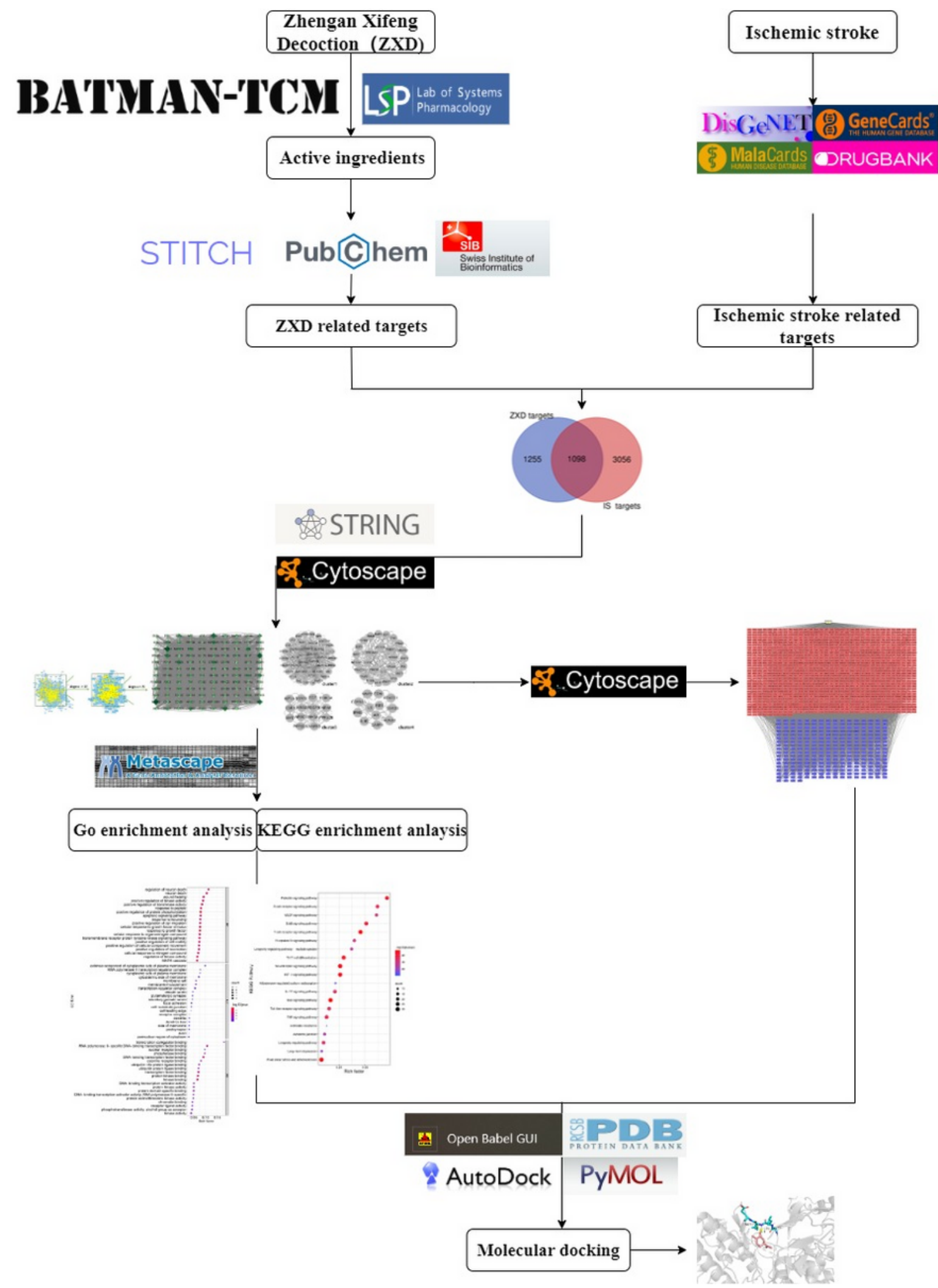

Figure 1

Flow chart of this article 
Figure 2

Venn diagram of intersection targets of ZXD and IS

Figure 3

PPI network (A total of 288 targets with degree $>13$ were obtained for the first time and 188 targets with degree $>30$ were obtained for the second time)

Figure 4

Sub-clusters of PPI network

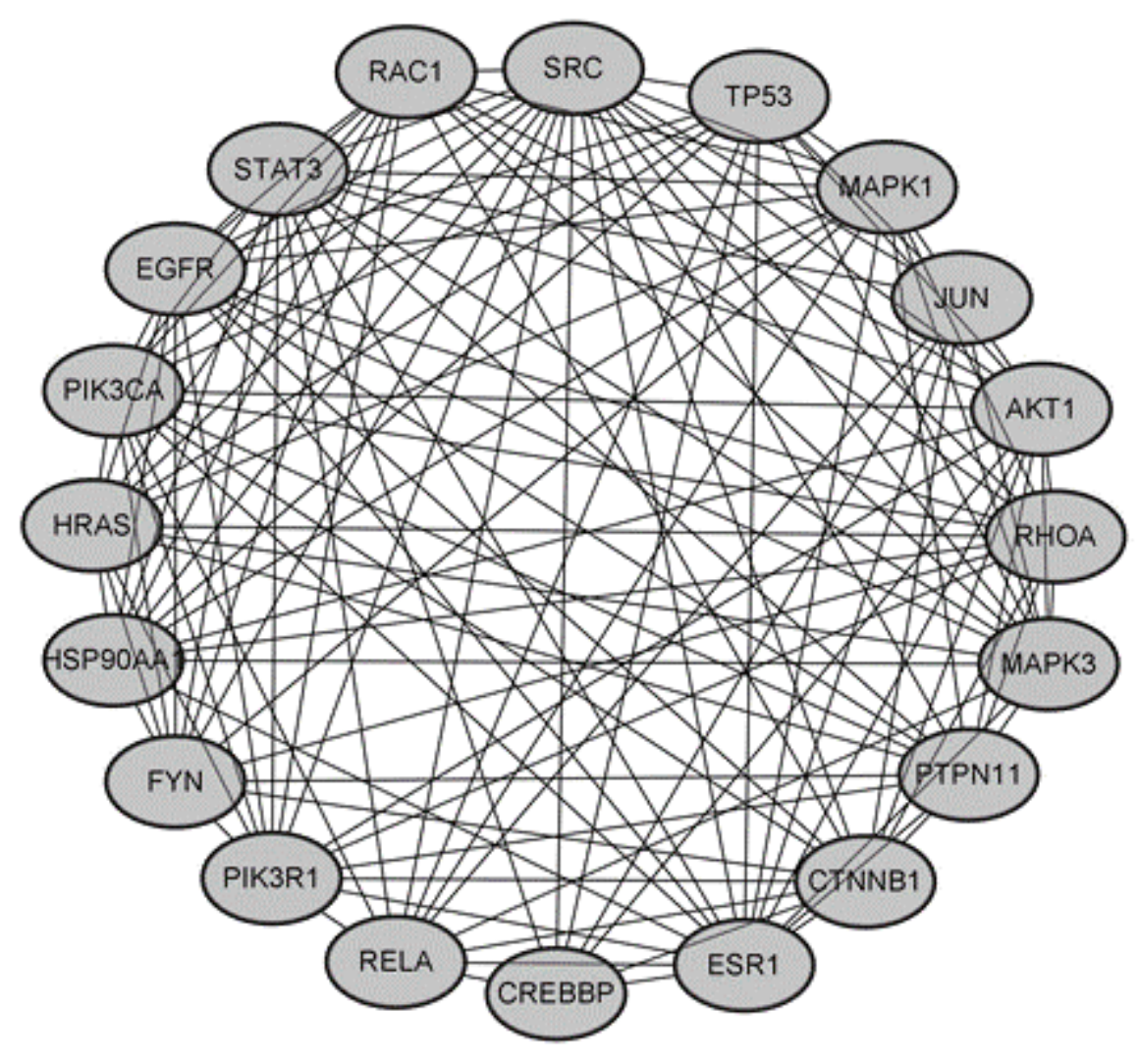

Figure 5

The 20 key targets of PPI network 


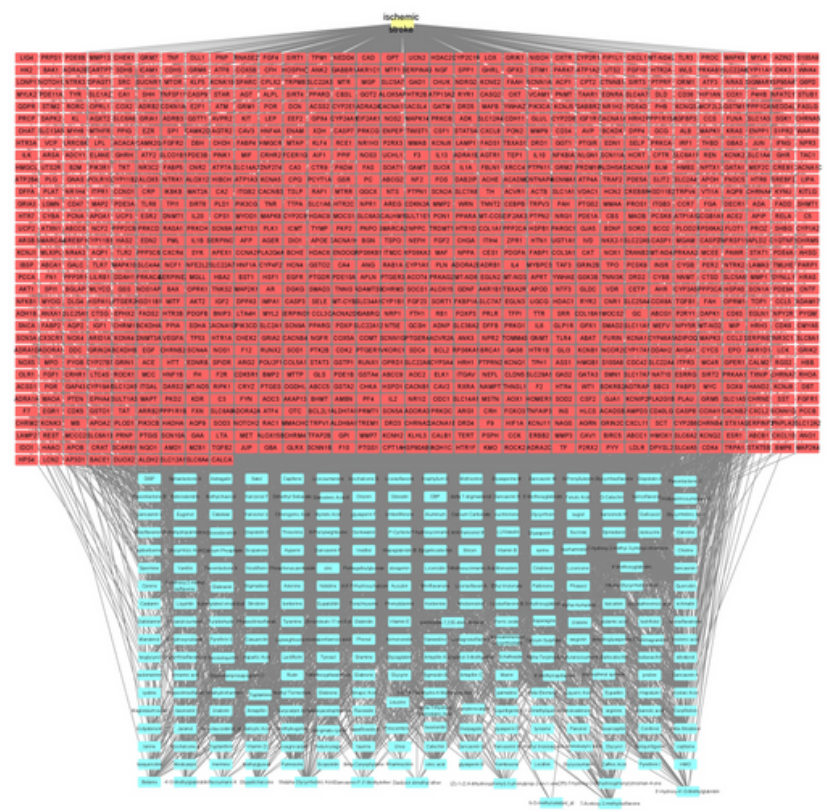

\section{Figure 6}

The ingredient-target-IS network (Red represents common targets, blue represents ingredients, and yellow represents diseases 


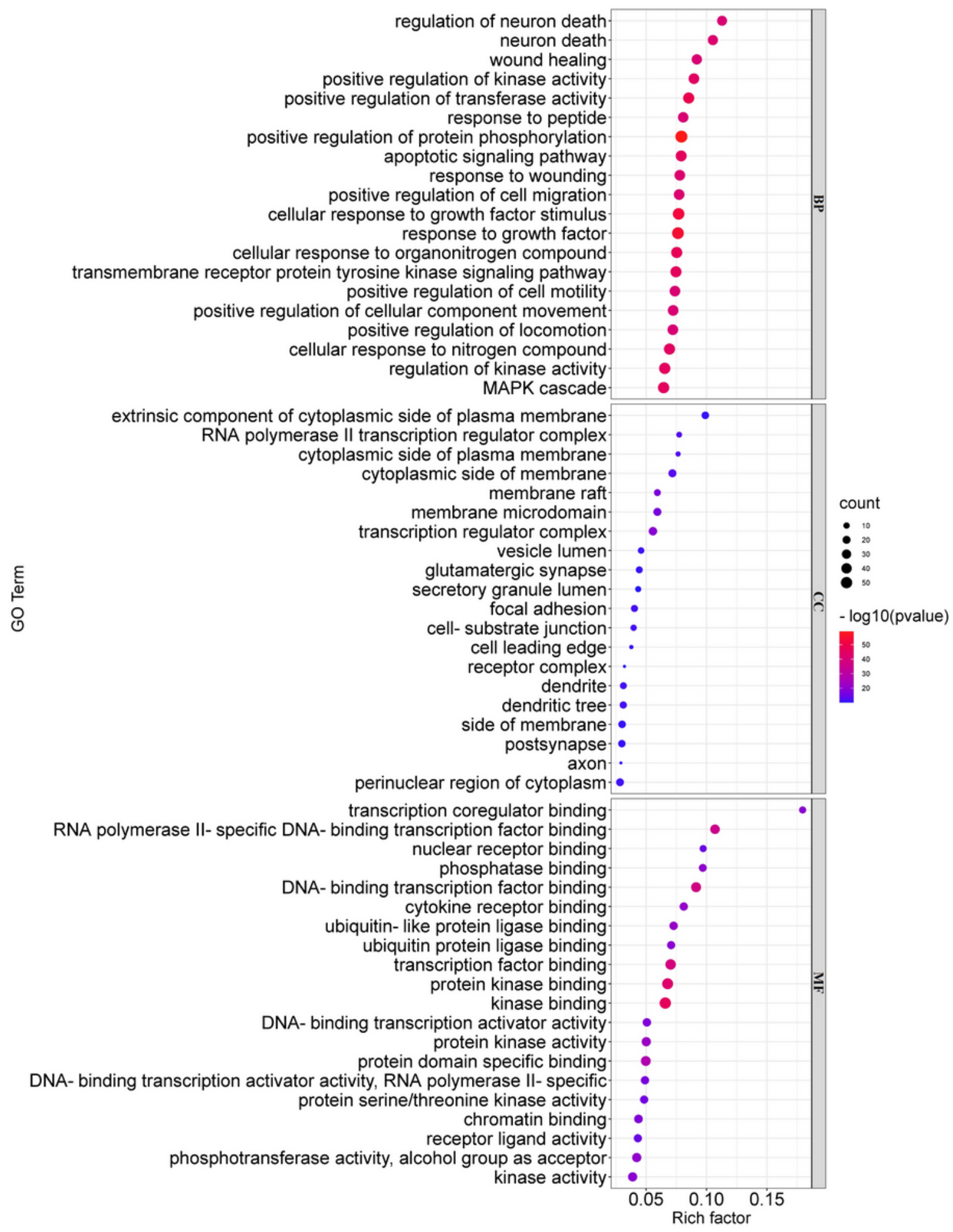

\section{Figure 7}

The top 20 terms of GO enrichment analysis of 118 core targets 


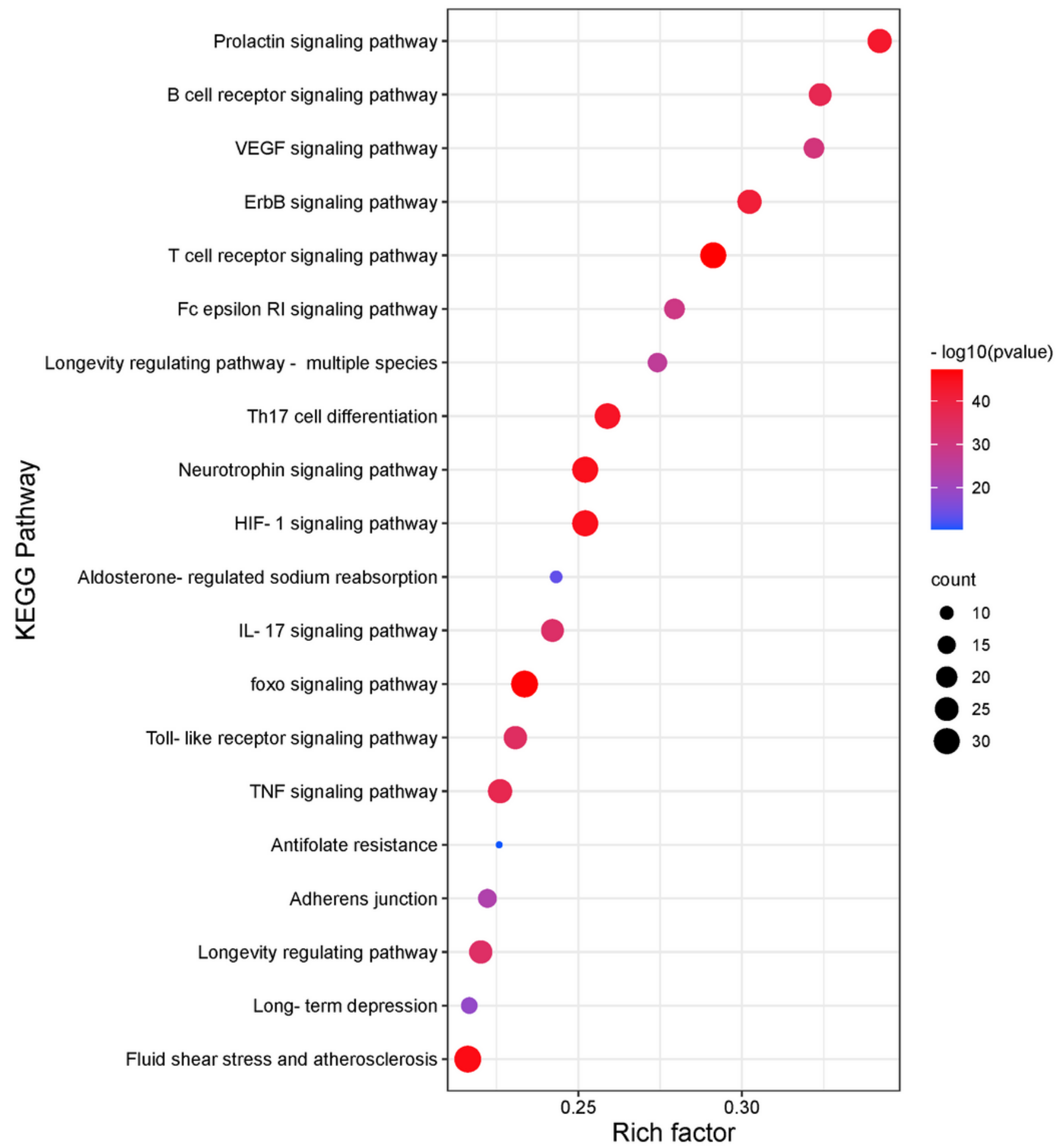

\section{Figure 8}

The top 20 terms of KEGG enrichment analysis of 118 core targets 


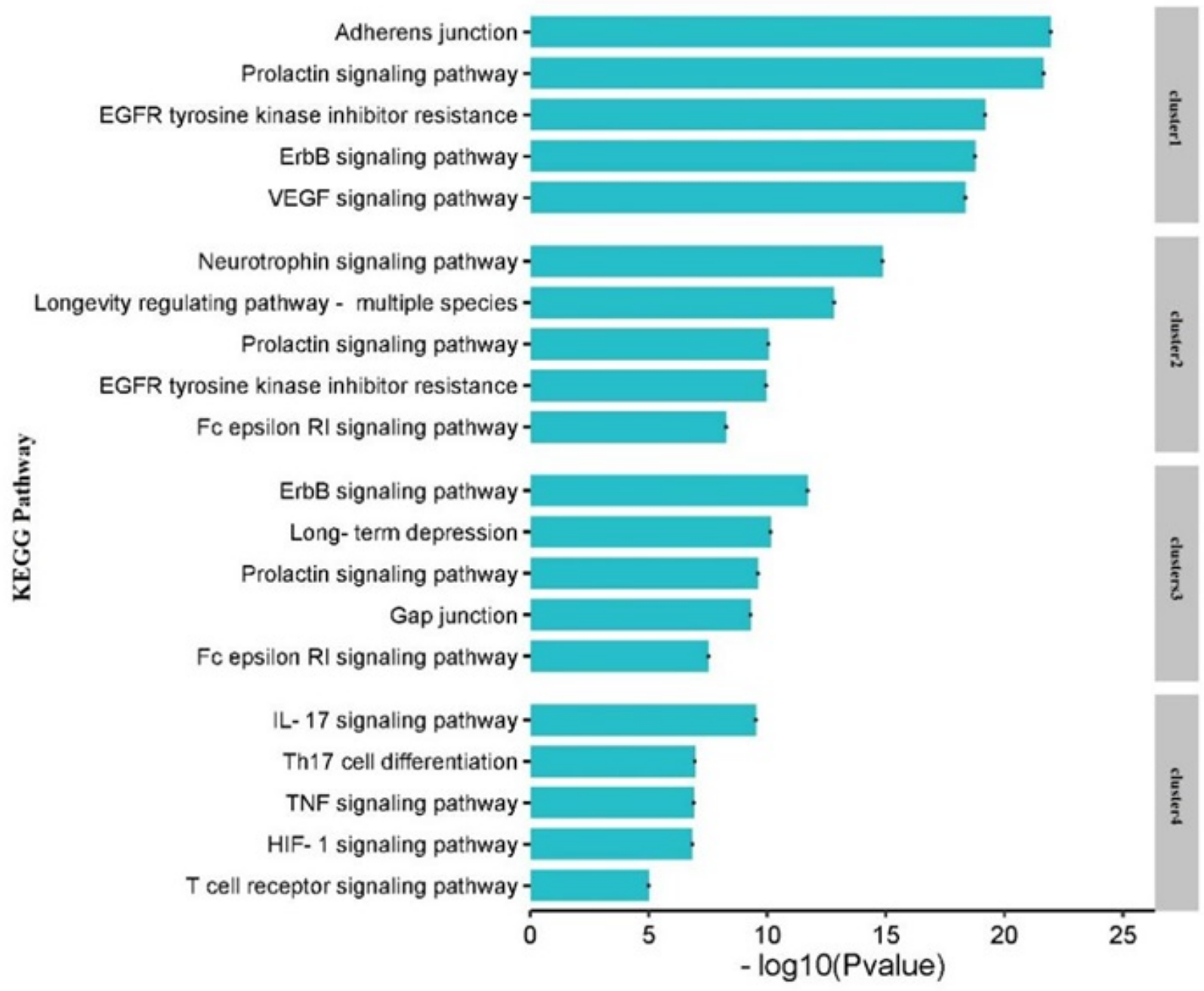

\section{Figure 9}

KEGG enrichment analysis of 4 sub-networks
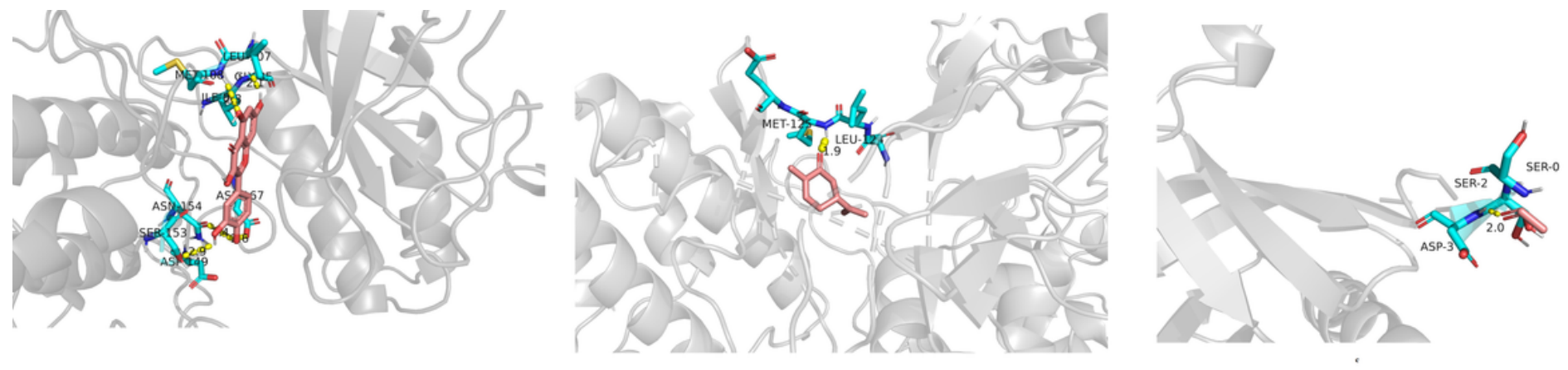

Figure 10

(a) The docking model of Quercetin with MAPK1 
(b). The docking model of Carvone with MAPK3

(c). The docking model of Asparamide with AKT1 